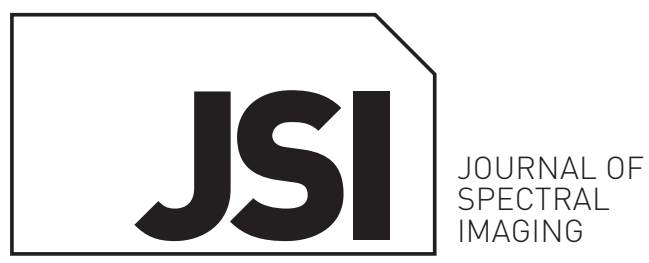

\title{
Exploring field-of-view non-uniformities produced by a hand-held spectroradiometer
}

\author{
Tamir Caras, ${ }^{a}$ Arnon Karnieli ${ }^{a,}{ }^{,}$and John Hedley ${ }^{b}$ \\ aThe Remote Sensing Laboratory, Jacob Blaustein Institutes for Desert Research, Ben Gurion University of the Negev, Sede Boker Campus, \\ 84990 Israel. Email: karnieli@bgu.ac.il
}

${ }^{b}$ Marine Spatial Ecology Laboratory, School of Biosciences, Hatherly Laboratory, University of Exeter, Prince of Wales Road, Exeter, Devon EX4 4PS, UK

\begin{abstract}
The shape of a spectroradiometer's field of view (FOV) affects the way spectral measurements are acquired. Knowing this property is a prerequisite for the correct use of the spectrometer. If the substrate is heterogeneous, the ability to accurately know what is being measured depends on knowing the FOV location, shape, spectral and spatial sensitivity. The GER1500 is a hand-held spectrometer with a fixed lens light entry slit and has a laser guide that allows control over the target by positioning the entire unit. In the current study, the FOV of the GER1500 was mapped and analysed. The spectral and spatial non-uniformities of the FOV were examined and were found to be spectrally independent. The relationship between the FOV and the built-in laser guide was tested and found to have a linear displacement dependent on the distance to the target. This allows an accurate prediction of the actual FOV position. A correction method to improve the agreement between the expected and measured reflectance over heterogeneous targets was developed and validated. The methods described are applicable and may be of use with other hand-held spectroradiometers.
\end{abstract}

Keywords: field of view (FOV), non-uniformity, GER1500, laboratory spectroscopy, Spectra Vista Corporation

\section{Introduction}

\section{At-issue}

Field spectroscopy is a key tool for the validation and development of remote sensing algorithms ${ }^{1-5}$ or estimation of chemical contents and properties in the environment. ${ }^{4-8}$ Field-collected end-member spectra are often assumed to represent the physical and chemical properties of a single (homogeneous) substrate, and are thus sometimes referred to as spectral signatures. ${ }^{5,6}$ Besides providing a quantitative measurement of purity, ${ }^{9-11}$ spectral signatures can form the basis for spectral unmixing analyses that are used to predict the partial contribution of a certain element within a heterogeneous substrate. ${ }^{12-15}$

The spectroradiometer, whether stationary or mobile, produces a spectral signal by recording the reflection of the substrate within its field of view (FOV), defined by the solid angle through which radiation enters the instrument. The FOV confines the circular area of surface measured by the instrument at a given distance. ${ }^{5}$ The homogeneity of the instrument's sensitivity across the FOV is often assumed to be spatially uniform. Unfortunately, the majority of commercial spectrometer manufacturers do not provide any technical details regarding FOV uniformity. Recent results demonstrate that the assumption of uniformity may not be correct and failing to fully characterise the FOV may result in errors caused by attributing spectral properties to the wrong target. ${ }^{16}$ The need to define the spectrometer's FOV prior to field or laboratory measurements may, therefore, be an important step before its use.

The homogeneity of a target is dictated by the scale ratio between the measured area and the size of the end-member units within it. If these units are small and evenly distributed, then measuring the heterogeneous substrate is less affected 
by FOV sensitivity. Conversely, as the scale ratio approaches the extent of the FOV. Deviations in FOV uniformity become significant sources of variation when, for example, attempting to estimate the combination of end-members or trying to detect a specific contributor within the substrate reflectance. While multiple random sampling provides unbiased mean estimations of reflectance of heterogeneous targets, knowledge of the FOV size is required to devise an adequate sampling strategy and to interpret the magnitude of variation around the mean. ${ }^{5-7}$

Another application in which assumed FOV uniformity is important is in the development and assessment of spectral unmixing methods. Sub-FOV quantification techniques, for example linear spectral unmixing or continuum removal, allow the investigator to estimate the end-member partial contribution within the mixed sample. ${ }^{12,17,18}$ However, predicting the linear contribution of end-members, for example, from spatially registered digital photography, ${ }^{19,20}$ is reliant on knowing the FOV's spatial and spectral sensitivity. Otherwise, non-uniformity will lead to inconsistency between the spectrally derived estimates and those based on the known proportions of the end-members in FOV. This would compromise the accuracy of assessment of the unmixing approach. For example, if an area of the FOV is under represented and the substrate is homogenous, then the error may be unnoticeable. If, however, in that area of the FOV the key end-member present in the sample is located, it will go unregistered. In another example, if the effective FOV is smaller than the expected FOV, one may miss out entire components of the sample. In any case, the level of heterogeneity and the size of components in comparison to the expected FOV make an important difference to the magnitude of the error.

FOV inaccuracies can be expressed as spatial non-uniformities in radiance detection obtained by the sensor and can be divided into three types:

Type A: the size and shape of the FOV-i.e. the shape of the area in which the sensor actually measures, compared to the shape or size of the area expected from the assumption of simple angular FOV specifications;

- Type B: measurement uniformity-i.e. how uniform is the intensity of light recorded from different positions within the FOV;

- Type C: spectral uniformity-i.e. how uniform is the sensor response throughout its spectral range.

MacArthur et al. ${ }^{16}$ studied two widely-used spectrometers that showed FOV distortions, mostly attributed to the spectrometer's light entry mechanism. Both of the tested spectrometers exhibited FOV deviations of all types listed above. In the tested Analytical Spectral Devices (ASD) FieldSpec Pro FR spectrometer, the fibre-optic bundle arrangement should have produced a more randomly scattered response function. However, the additional $10^{\circ}$ lens produced a rather patchy FOV with a few "blind" spots where no light sensitivity is recorded. ${ }^{16}$ In the fixed focus lens GER3700, manufactured by Spectra Vista Corporation la wider wavelength range version of the GER1500), the response function testing showed a strong shape effect due to the entry slit aperture and lens that implies that these distortions may be unit specific. While the results described by MacArthur et al. ${ }^{16,21}$ are detailed, their methodology requires precise light source positioning equipment and a substantial investment of investigator time. The present study was conceived out of the need for a more rapidly implemented FOV assessment scheme that could be applied prior to laboratory studies and field work.

The current study generally aims to describe the performance of the GER1500 spectroradiometer with regards to its FOV properties. The specific objectives were to: (1) quantify the non-uniformity of the sensor in terms of the difference between the expected and the actual measured areas laddressing Type A, B and C distortions); (2) develop a method for correcting the non-uniformity; and (3) establish the relationship between the fitted laser guide position and the effective FOV. Besides being simple to undertake, the method developed here offers the users an opportunity to be familiarised with their unit's FOV before undertaking fieldwork. It should be noted that the proposed method is applicable for any field or laboratory spectroradiometer and not exclusive to the GER1500.

\section{Basic assumptions}

Before attempting to describe the FOV of the GER1500, the following assumptions, common to all spectrometers and for which all further spectral measurements were relied upon, were made:

- the viewed surface is flat li.e. would produce a lambertian reflection);

avery point within the FOV has the same reflectance intensity as any other point (i.e. FOV is even and there is no patchiness in reflection intensity);

the amount of reflected radiation from a sub-area within the FOV is proportional to the reflected radiation from the entire FOV (i.e. a sample of $1 \%$ of the FOV area should reflect $1 \%$ of the radiation reflected by the entire FOV);

- the reflectivity of the "black background" (equivalent to dark current) is extremely low and negligible;

- the "white reference" panel is perfect li.e. reflects all light arriving at it).

\section{Methodology}

The current study was carried out in three steps: (1) mapping the FOV and developing a non-uniformity correction technique; (2) validation of the correction method; and (3) describing the relationship between the FOV and the laser guide.

\section{Experimental design}

For the current research, the GER1500 spectrometer manufactured by Spectra Vista Corporation was used. It is an allin-one unit with internal memory that supports independent operation. It offers a spectral range between $300 \mathrm{~nm}$ and $1100 \mathrm{~nm}$ with a spectral resolution of $3 \mathrm{~nm}$. For the purpose of the current experiment, only the spectral range of between 


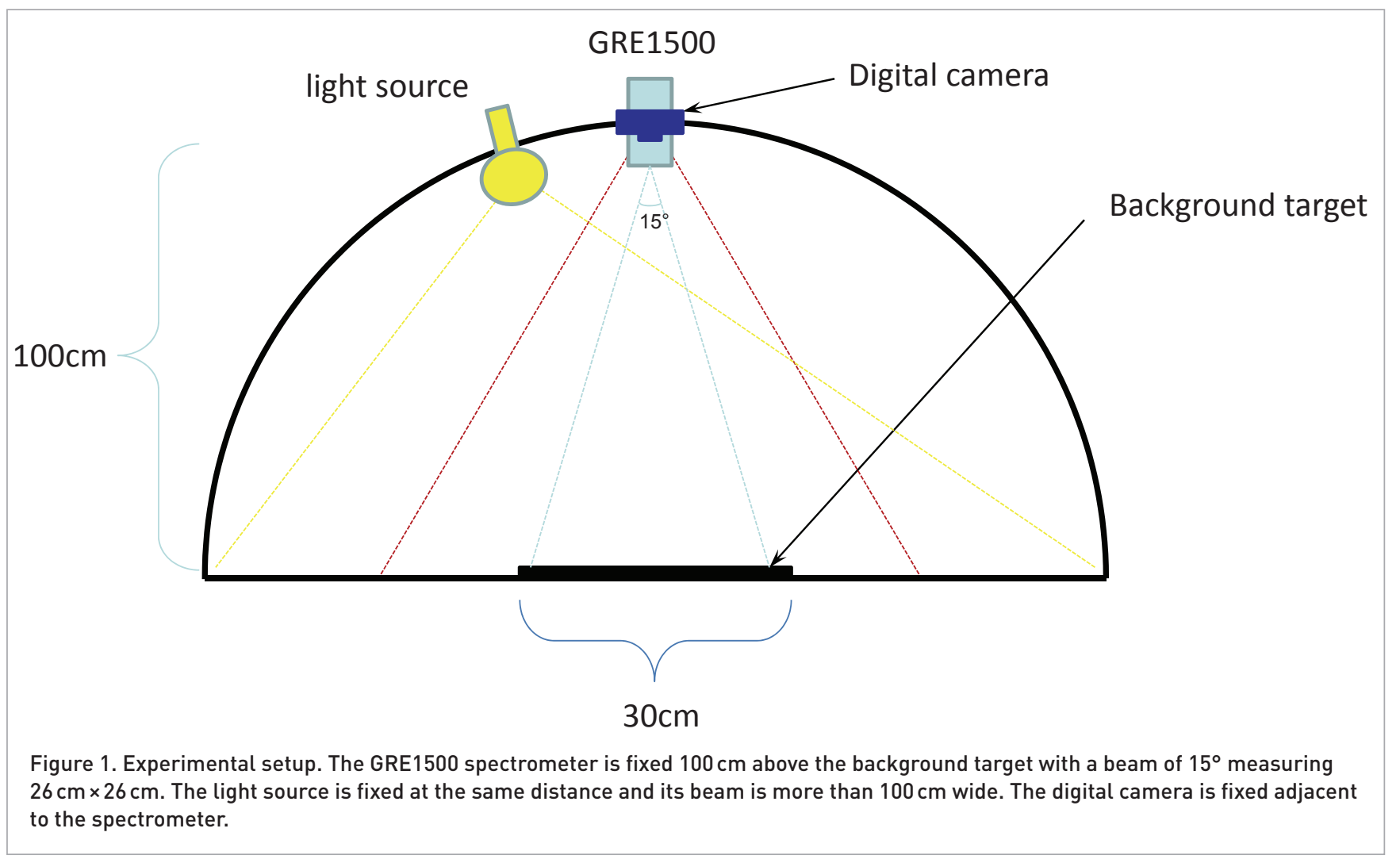

$400 \mathrm{~nm}$ and $700 \mathrm{~nm}$ was used. Its fixed lenses and laser guide allow highly accurate pointing, albeit offset from each other. The spectrometer was fitted with a standard $15^{\circ}$ lens and mounted on a construction giving a constant distance of $1 \mathrm{~m}$ from the target (Figure 1). These dimensions correspond to a $26.3 \mathrm{~cm}$ target diameter. The experimental light source was a standard outdoor halogen lamp (250 W pro-light, P2-10CE manufactured by Lowell mounted at $72^{\circ}$ to the experimental plane $\left(18^{\circ}\right.$ zenith angle). The light provided a $1 \mathrm{~m}$ wide beam on the experimental plain and was assumed to be uniform across the strip plain. A digital camera was mounted in parallel to the spectrometer to provide a photographic record for each measurement. The spectrometer unit, digital camera and the illumination remained fixed in their position for the entire duration of the study.

The target background on which all experiments have taken place was a $30 \mathrm{~cm} \times 30 \mathrm{~cm}$ wooden surface covered by black velvet-like material. It reflected less than $2 \%$ of the Halon plate used as white reference. The grids mentioned from here on were marked by pressure marks on the edges of this surface and provided virtual lines for guidance. The measured target was a $2 \mathrm{~cm} \times 2 \mathrm{~cm}$ white Halon plate that was placed on the background. The plate was $1.2 \mathrm{~mm}$ thick and the edge shadowing was assumed negligible. The FOV mapping was performed in two stages. In the first stage of the experiment, the Halon plate was moved along from the upper-right corner to the lower-left corner on a pre-determined $28 \mathrm{~cm} \times 28 \mathrm{~cm}$ grid [Figure 2(a)]. This process provides a rough definition of the entire FOV as expected from the sensor with a $15^{\circ}$ angle lens at $1 \mathrm{~m}$ distance. The grid map was used to identify regions of high or low sensitivity within the FOV. The second stage focused on a strip of $3 \mathrm{~cm} \times 23 \mathrm{~cm}$ within the expected FOV [Figure $2(b)$ ]. This time, the procedure was repeated with a smaller $(1 \mathrm{~cm} \times 1 \mathrm{~cm})$ white Halon plate. Subtracting the black reference from each reading gave the final "per pixel" contribution in percentage (linearity was assumed). Dividing each individual reflectance reading by the total reflection (i.e. the summation of the reflection of the 69 sequential positions of the Halon plate on the grid) provides its actual, spatial specific, partial contribution.

The final product of the mapping stages is a raster image consisting of the reflectance values of all the grid points within the strip. The resulted image was exported into ENVI software (http://www.ittvis.com/) ready to act as a wavelength specific mask [Figure 3(a)]. In this mask, each pixel, at each wavelength, had a designated sensitivity weight. In contrast to the first and second assumptions, these pixels were not equal in value but cumulatively they produced $\sim 100 \%$ of the FOV reflection. Moreover, from here on, the effective FOV area is often referred to as the strip.

White reference measurements were obtained before each reading while black reference measurements were repeated periodically. It is important to note that all raw spectral readings were subjected to calibration supplied with the unit. Both showed insignificant variance throughout the experiments. The black background was used as "zero" reflectance marker 
(a)

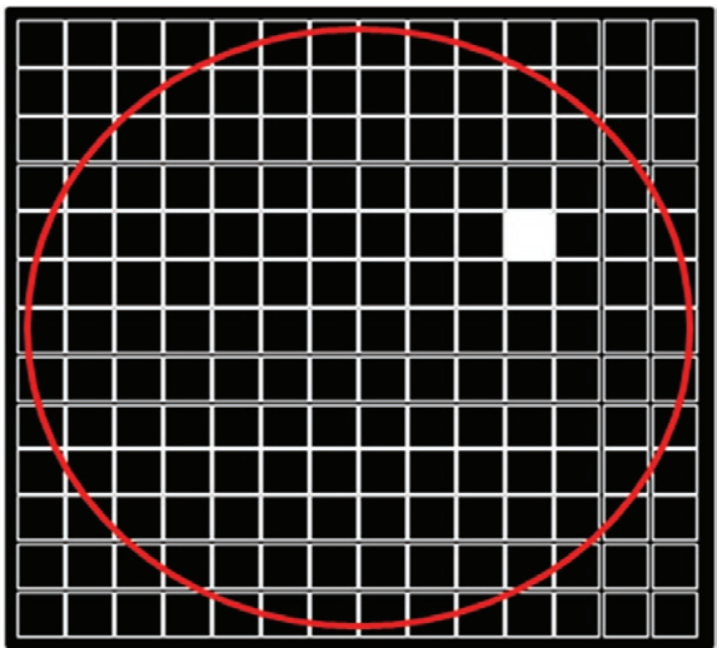

(c)

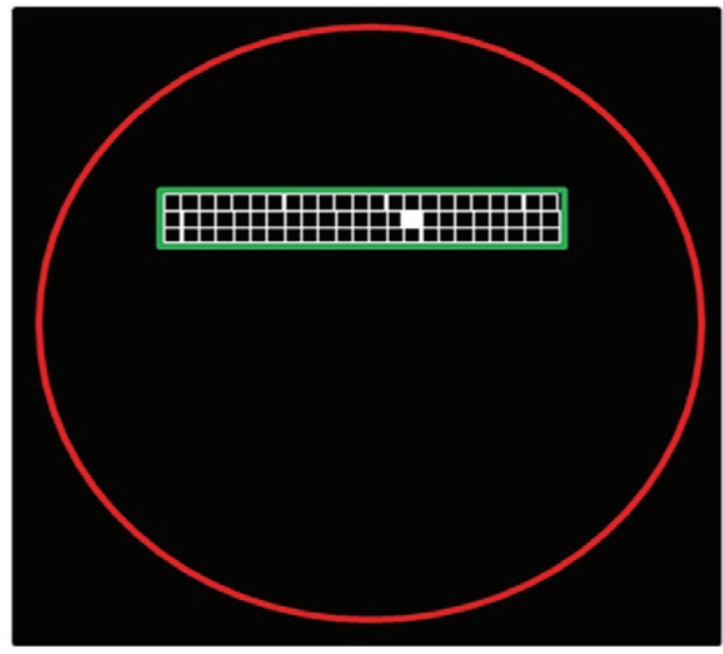

(d)

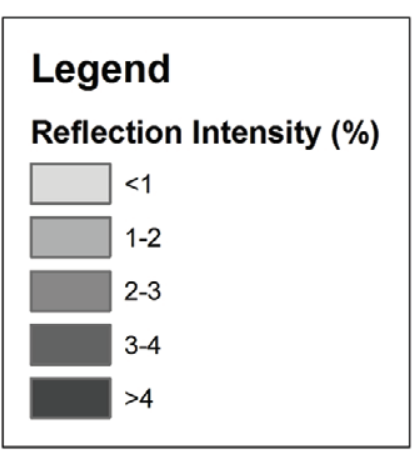

(b)

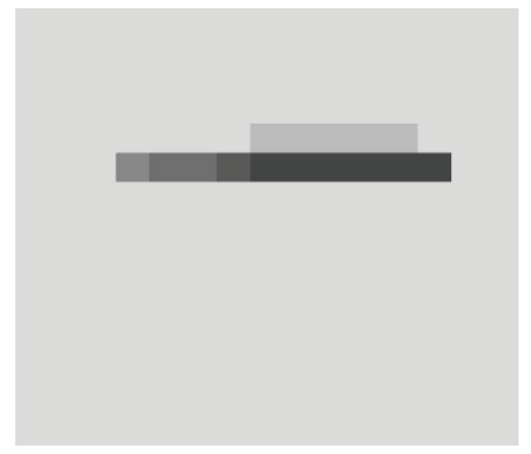

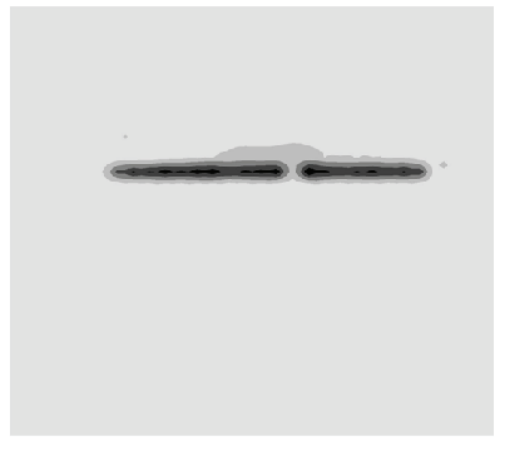

Figure 2. The expected field-of-view (the dark area within the red circle) was mapped twice in two spatial resolutions-first at $2 \mathrm{~cm} \times 2 \mathrm{~cm}$ and then $1 \mathrm{~cm} \times 1 \mathrm{~cm}$ using a cut-out piece of white plate. (a) A $2 \mathrm{~cm}$ plate was moved around the black reference, eventually covering the entire area, creating a complete grid of $26 \mathrm{~cm} \times 26 \mathrm{~cm}$; (b) the actual sensitivity per "pixel" of the field of view after the first mapping stage using the $2 \mathrm{~cm} \times 2 \mathrm{~cm}$ plate. Darker shades represents high sensitivity while the generally light grey represents $<1 \%$ sensitivity; (c) the apparent FOV strip (green rectangle) within the expected FOV (red circle), was mapped using the same technique; (d) the interpolated sensitivity of the field of view after the second mapping stage using the $1 \mathrm{~cm} \times 1 \mathrm{~cm}$ plate. Notice the "blind spot" artifact at the centre of the strip which is probably attributed to a physical malfunction of the sensor array.

and was deducted from each reading laccepting assumption 3). The "white reference" and plates were made from the same material and were spectrally identical to an industrial standard (a Spectralon panel by Labsphere) within the relevant spectral range.

\section{Mixing correction and validation}

To determine how well the correction mask works, an experiment using mixed coloured targets was designed. Reference samples made of four coloured foams li.e. red, green, blue and yellowl were arranged in the target measuring area, covering the FOV strip area. Because the FOV strip is not uniform in its light sensitivity, the spectroradiometer is likely to record somewhat different combination of colours than their real colour in reality. The correction mask is expected to rectify this error and the difference between the "actual" and "corrected" image results is used to quantify the correction success.

The foams were placed at three different orientations [Figure 4): (1) two foams arranged vertically (lengthways) in $3 \mathrm{~cm}$ wide bands [Figure 4(a)] in different colour combinations (red-green, red-blue, blue-green); (2) three large foams arranged horizontally in six different colour combinations [Figure 4(b)]; and (3) four small foams arranged horizontally in eight different colour combinations [Figure 4(c)]. Note that the foam sizes were not equal and, therefore, the digital camera operating simultaneously was used to determine the partial contribution of each colour in each set (percentage cover of the strip). Additionally, 


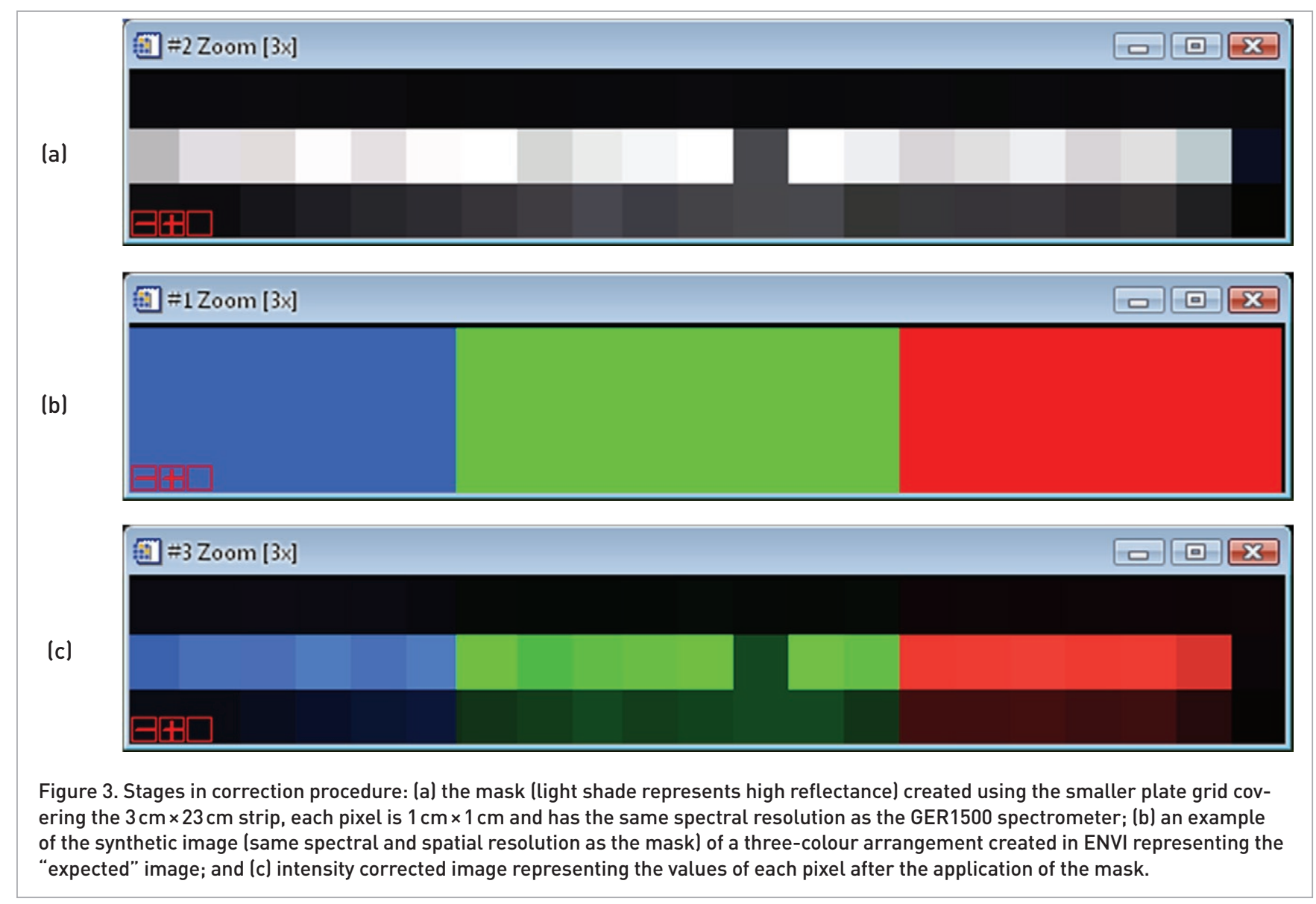

since the foam pieces were arranged one next to the other they formed a continuous surface and no shadowing occurred.

Readings of each arrangement with the GER1500 provided the sensor's "actual" spectra. As the spectrometer records only the total reflection from the entire strip it cannot be corrected, so a synthetic image identical in size to the strip was created by ENVI software. Unlike the spectrometer reading, the synthetic image contains $69(23 \times 3)$ pixels of $1 \mathrm{~cm}^{2}$ each and is therefore of identical dimensions as the mask. Pure hyperspectral spectra of each colour substrate provided the necessary endmembers for the synthetic image production. The resulting synthetic image was a hyperspectral version of the image taken by the digital camera [Figure 3(b)], spectrally compatible with the spectrometer's spectral resolution. Averaging all
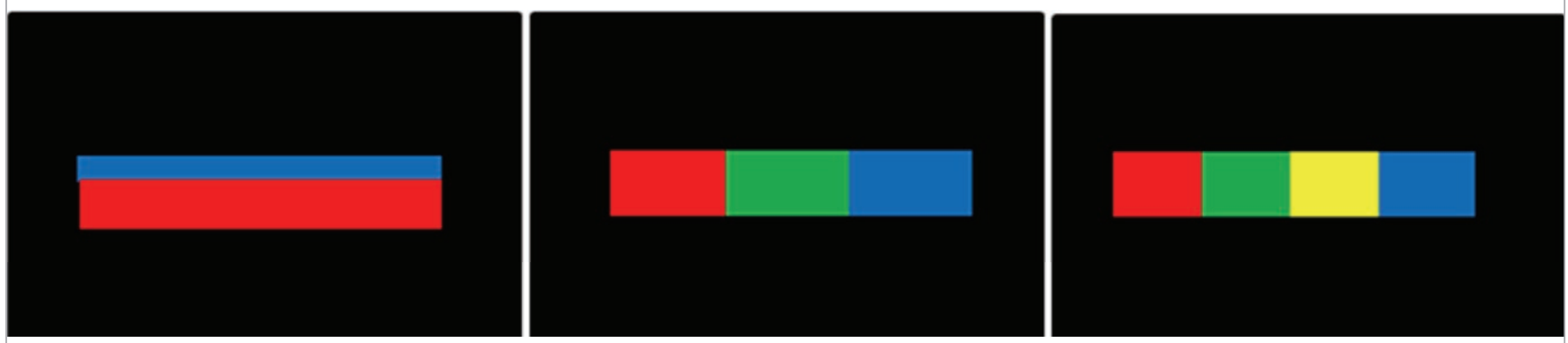

Figure 4. Colour foam arrangements on the black background. (a) Vertical arrangement. As the colour foam bands were $3 \mathrm{~cm}$ wide, the first and last of the four readings had a "pure" single colour reflectance; (b) larger horizontal three-colour arrangements were made of $8 \mathrm{~cm}$ long pieces; and (c) Smaller horizontal four-colour arrangements $-6 \mathrm{~cm}$ long. Note that in both horizontal arrangements one colour was always $1 \mathrm{~cm}$ out of bounds. 
the pixels in the synthetic hyperspectral image produced the mixed spectrum that is analogous to the one recorded by the spectroradiometer. It assumes uniform FOV sensitivity, giving each coloured pixel an equal intensity weight. This spectrum was termed "expected" as it is expected to produce-assuming linear mixing - a spectrum identical to that of the GER1500. At the correction stage, the synthetic image was multiplied by the mask-pixel-by-pixel and wavelength-by-wavelength [Figure 3(c)]. The averaging of the resulting image, where each pixel is weighted differently, gave the final product, i.e. the "corrected" spectrum. The above procedure was repeated for every colour substrate combination (17 in total) that allowed a comparison between the "expected" spectra, the "corrected" spectra and the "actual" spectra.

\section{Accuracy estimation}

In this final step, spectra for each colour arrangement were compared and the similarity indicated how well the correction procedure performed. The two produced spectra ("expected" and "corrected") were compared to the "actual" spectrum using two complementary methods: a linear regression model $\left(r^{2}\right)$ and the root mean squared error (RMSE).

\section{Laser guide position}

Ideally, a field operator knows where the approximate location of the actual FOV area is in space. The GER1500 is an independent hand-held spectrometer with a set opening slit and a fixed laser guide. The position of the FOV strip is expected to be linearly correlated to the laser guide position because the angle between the slit and the laser guide is fixed in the unit. If this relationship is known in advance, the operator can achieve high pointing accuracy. To confirm this relationship, the size and location of the strip was measured at four distances from a target $(17 \mathrm{~cm}, 57 \mathrm{~cm}, 150 \mathrm{~cm}$ and $210 \mathrm{~cm})$ and plotted in a regression function against the laser guide position.

For this purpose, a large (A3 size, $420 \mathrm{~mm} \times 297 \mathrm{~mm}$ ) matt black card was hung on a wall. The spectroradiometer was fixed and carefully levelled on a table so it was directed at the centre of the card at the zenith angle. Knowing the approximate dimensions and shape of the FOV strip from previous steps described above, a white reference card was moved in four vertical and horizontal directions in $1 \mathrm{~cm}$ increments, starting at the laser guide point. Any spot where the white reference registered a change from the expected "black reference" (close to zero reflectancel was marked as "within the strip". The outer borders of the strip were defined as the point from which the white reference placement did not register in the instrument reading (thus outside the strip). Finally, a linear relationship between the approximated strip and the laser guide was calculated and its accuracy $\left(R^{2}\right)$ evaluated (Figure 5).

\section{Results}

As a result of moving the $2 \mathrm{~cm} \times 2 \mathrm{~cm}$ plate over the entire expected FOV $(26 \mathrm{~cm} \times 26 \mathrm{~cm})$, it was found that the actual reflection area is much smaller than the expected FOV demonstrated in Figure $2(b)$. Almost all the reflected radiation was concentrated in a strip of $3 \mathrm{~cm} \times 23 \mathrm{~cm}$ [Figure 2(c)] that was located vertically and to the right of the spectrometer expected plane. The matrix of plate reflection was plotted for normal distribution and randomness. Distribution results (not presented here) showed a strong bi-model shape where all the area around the strip was normally distributed and random across the experimental plane. The area around the strip was also normally distributed.

The peripheral plates (those outside the strip) contributed between $0 \%$ and $0.1 \%$ of the total reflectance for the entire area while within the strip, plates ranged between $1 \%$ and $9 \%$ of the total. This difference in a magnitude of ten makes a clear strip shape within the expected FOV. The strip described is only $12 \%$ of the area the instrument is expected to read. Next, in order to describe the reflected area more precisely, the $1 \mathrm{~cm} \times 1 \mathrm{~cm}$ was moved within the strip [Figure 2(c)]. This refinement confirmed the very strong concentration of reflectance that is responsible for $97 \%$ of the entire radiance arriving at the sensor [Figure 2(d)]. In actuality, the area responsible for $97 \%$ of measured radiance is only about $5 \%$ of the expected FOV area. Except for differences in very low magnitude, it seems that this non-uniformity does not change throughout the spectral range (Figure 6).

As shown in Table 1, comparing the "corrected" spectra with the "actual" spectra produced much better matches than the comparison between the "expected" spectra and the "actual" spectra (please note that each spectrum represents an averaged synthetic image). This result is demonstrated as $r^{2}$ is higher in 18 out of 20 cases and RMSE scores are higher in 19 out of the same 20 cases. The comparisons highlighted a clear error in vertical colour bands [Figure $4(a)$ ]. In comparison to vertical colour bands, horizontally distributed colour mixtures of three or four colours [Figures $4(b)$ and $4(c)$ ] were generally less different than the "actual" spectra. In the three-colour arrangements, the difference between the "expected" and "actual" were intermediate (average $r^{2}$ is 0.87 and RMSE 0.96). The similarity between "actual" and "corrected" output was slightly higher (average $r^{2}$ is 0.97 and RMSE 0.98) than that of "actual" and "expected". The "expected" and "corrected" comparison scores for the four-colour arrangements were generally higher than those of the three-colour arrangements, indicating negative correlation to number of endmembers in the mix. Note that all $r^{2}$ correlation results are statistically significant (i.e. $p<0.05$ ). In addition, the results for $r^{2}$ are spread across a wider range li.e. 0.06 to 0.99), while the RMSE values range from 0.83 to 0.99 . The relationship between the two different score systems is not strong (linear regression $r^{2}=0.65$ ).

Limiting the spectral range for the used spectra highlights the difference between using the $r^{2}$ and the RMSE as a measure of difference. The degree of similarity between "actual" and "expected" spectra was heavily impacted by the spectral restriction when using the $r^{2}$ method [Figure 7(a)]. 


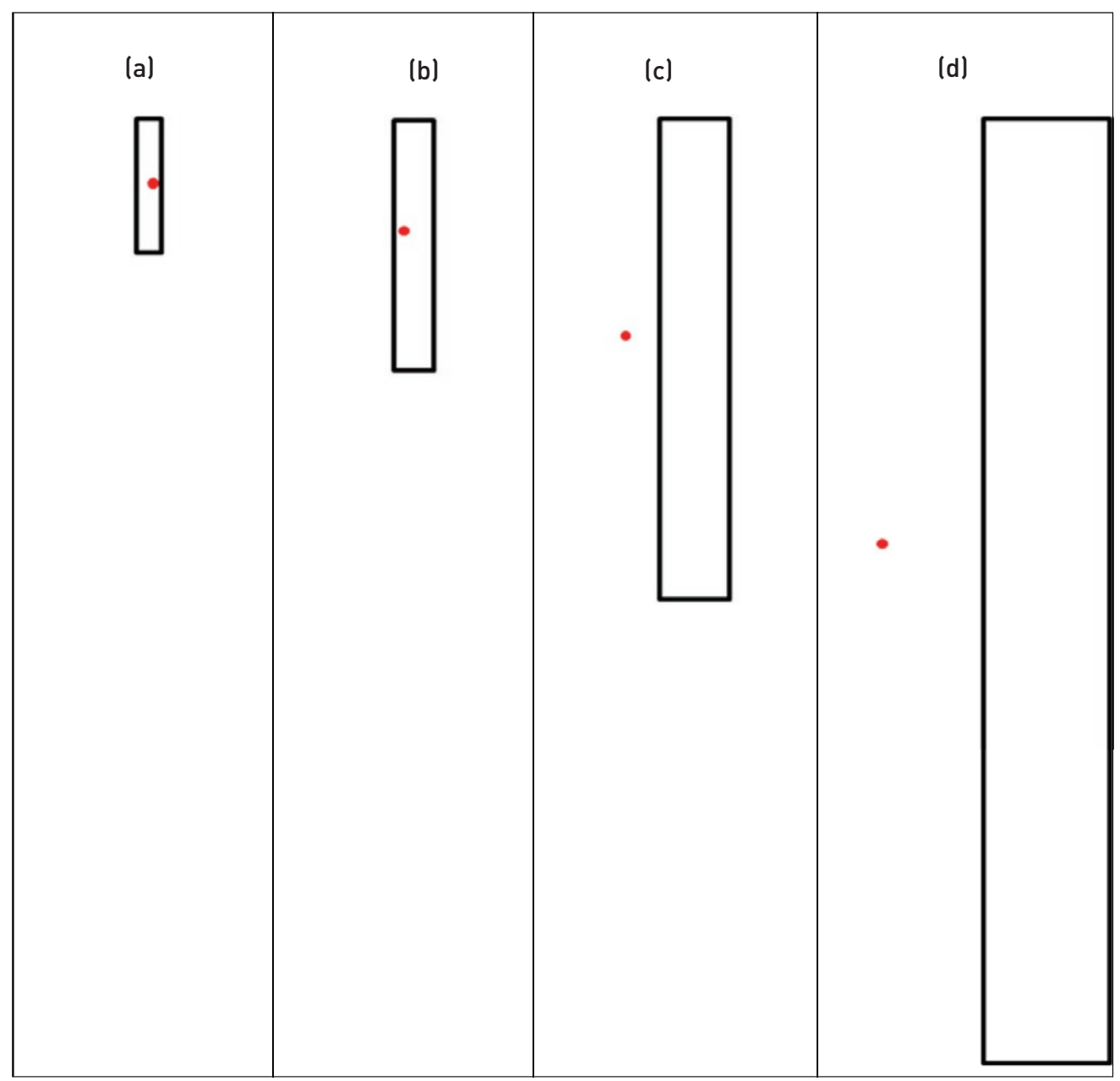

Figure 5. Strips and laser guide position. The position of the laser guide in relationship to the real FOV is a function of distance from the sensor. Here are four different distances $[50 \mathrm{~cm}, 100 \mathrm{~cm}, 150 \mathrm{~cm}$ and $200 \mathrm{~cm}-(\mathrm{a}),(\mathrm{b}),(\mathrm{c})$ and (d), respectively) and their relative position to the laser guide. Laser guide position is the red dot and corresponding to the strip position (black rectangle). Up to a meter distance - the laser guide is within the FOV strip. At distances longer than $1 \mathrm{~m}$ the actual FOV strip drifts right of the laser guide.

Conversely, when using the RMSE comparison, the results remain relatively constant as the range decreases [Figure $7(b)]$.

As shown in Table 2, strip sizes increase linearly with distance. The FOV strip position shows a linear relationship with the position of the laser guide (Figure 5).

\section{Discussion}

The mapping of the FOV was consistent at both resolution levels used $(2 \mathrm{~cm}$ and $1 \mathrm{~cm}$ pixel size). The higher resolution mapping $(1 \mathrm{~cm} \times 1 \mathrm{~cm})$ provided a good, wavelength dependent correction mask that was used successfully for all three colour arrangements. The higher similarity between the corrected

Table 1. Grouped and averaged results for linear regression $\left(r^{2}\right)$ and $R M S E$ at three-colour set-ups (Figure 3). Because the RMSE system of scoring is affected by light intensity, a normalised RMSE version was tried and its scores can be compared with both the regular RMSE and the $r^{2}$ scoring systems. Normalised RMSE scoring is noticeably similar in values to $r^{2}$ as well as the average difference between the expected and corrected results.

\begin{tabular}{|l|c|c|c|c|c|c|}
\hline \multirow{2}{*}{} & \multicolumn{2}{|c|}{ RMSE } & \multicolumn{2}{c|}{ RMSE normalised } & \multicolumn{2}{c|}{$r^{2}$} \\
\cline { 2 - 7 } & Expected & Corrected & Expected & Corrected & Expected & Corrected \\
\hline Average 2 colour (top to bottom) $n=3$ & 0.88 & 0.91 & 0.63 & 0.93 & 0.63 & 0.95 \\
\hline Average 3 colour (horizontal) $n=6$ & 0.96 & 0.98 & 0.90 & 0.96 & 0.86 & 0.97 \\
\hline Average 4 colour (horizontal) $n=8$ & 0.95 & 0.98 & 0.92 & 0.97 & 0.94 & 0.99 \\
\hline
\end{tabular}



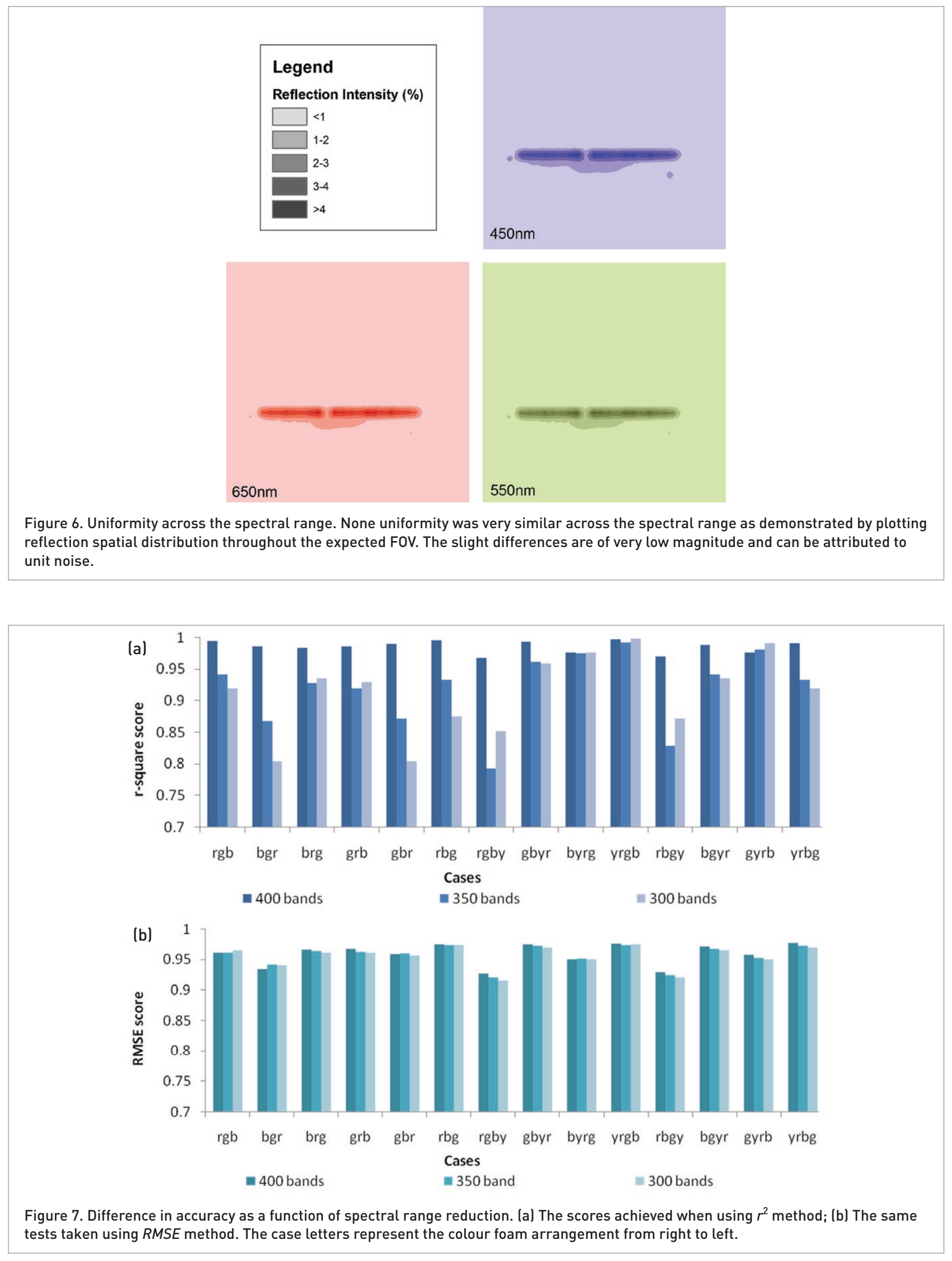
Table 2. A linear increase in size of the FOV strip with distance. $r^{2}$ represents the accuracy of the linear relationship and linear regression equation models the shift (bottom of each column).

\begin{tabular}{|c|c|c|c|c|}
\hline $\begin{array}{c}\text { Distance from } \\
\text { GER1500 } \\
(\mathbf{c m})\end{array}$ & $\begin{array}{c}\text { Height of strip } \\
\text { (cm) }\end{array}$ & $\begin{array}{c}\text { Width of strip } \\
\text { (cm) }\end{array}$ & $\begin{array}{c}\text { Distance of laser } \\
\text { from strip top } \\
\text { (cm) }\end{array}$ & $\begin{array}{c}\text { Distance of laser from } \\
\text { strip centre (to the left) } \\
\text { (cm) }\end{array}$ \\
\hline 17 & 5.69 & 1.95 & 2.55 & -1.56 \\
\hline 50 & 12.65 & 2.76 & 5.69 & -0.20 \\
\hline 100 & 23.20 & 3.98 & 10.45 & 1.85 \\
\hline 150 & 33.74 & 5.21 & 15.20 & 3.91 \\
\hline 200 & 44.29 & 6.43 & 19.96 & 8.96 \\
\hline 250 & 54.83 & 7.66 & 24.71 & 10.07 \\
\hline 300 & 65.38 & 8.88 & 29.47 & 12.13 \\
\hline 350 & 75.92 & 10.11 & 34.22 & 14.18 \\
\hline 400 & 86.47 & 11.33 & 38.98 & 16.24 \\
\hline 450 & 97.01 & 12.56 & 43.73 & 18.29 \\
\hline 500 & 107.56 & 13.78 & 48.49 & - \\
\hline$r^{2}$ & 1.00 & 1.00 & 1.00 & $Y=0.04 X-2.25$ \\
\hline Regression* & $Y=0.21 X+2.10$ & $Y=0.02 X+1.53$ & $Y=0.09 X+0.93$ & \\
\hline
\end{tabular}

${ }^{*} Y$ is the expected value and $X$ is the distance in $\mathrm{cm}$

and the actual readings suggest that the correction is working well and, therefore, that the non-uniformity is consistent with the FOV mapping. The effect of the non-uniformity is more visible in the two-colour bands because these are paralleled to the slit direction. This would result in higher under/over representation as two of the colour bands are always in an "under represented" part of the FOV strip. In the three- and fourcolour arrangements, the non-uniformity is reduced as the colour targets are generally equally measured within the FOV strip. Additionally, as more colours are arranged on the FOV strip, the mix becomes effectively more homogeneous and thus less affected by the non-uniformity. The results demonstrated here show an element of this when the differences between "expected" and "actual" spectra are compared for four- and three-colour mixes.

The results described in this study demonstrate how the FOV of the GER1500 is spatially biased and non-uniform. This seems to closely follow the instrument's opening slit and is wavelength independent. Previous studies with a different spectrometer model (GER3700, by the same manufacturer) have established a detailed and similar directional response function distortion. ${ }^{16,21}$ Their results show how the FOV of the visible and near infrared sensor hosts a sensitivity hotspot similar in shape and location to the one demonstrated here. However, their superior mapping quality is not easy to repeat without intensive laboratory work and dedicated equipment. The method described here offers a simple solution, forming an a priori knowledgebase for the FOV of a specific unit. It can be undertaken in any laboratory or potentially even under field conditions.

When the end-members in measured targets are not distributed homogeneously, a single spectral reading in its uncorrected state may lead to errors in the analysis. For example, if parts of the subject of interest are located outside the GER1500 sensitive strip, they are likely to contribute much less than expected to the final spectra (i.e. severely under represented). This scenario is expected to be very common, considering that only about $5 \%$ of the expected FOV contributes to the final spectrometer's reading. The error we describe is directly related to the physical construction of the GER li.e. fixed lens and light entry slit and fixed internal optics) and thus unlikely to be applicable to sensors with fibre-optic light entry [for example, Ocean Optic sensors, Analytical Spectral Devices (ASD) and GER1500 with a fibre-optic adaptor]. An unmasked fibre-optic bundle randomly distributes its sensitivity across the FOV but may produce a different type of non-uniformity. ${ }^{21}$ Therefore, because the fine tuning correction method relies on a known and fixed relationship between the light entry and the spectrometer, it is unsuitable for a fibre-optic bundle where the light entry is randomised by the fibre-optic bundle. However, the method of mapping the FOV properties is universal and can be adapted for any spectrometer (even if a fibre-optic is used) as a precursor step before designing future sampling strategy.

There is no need to correct the FOV if the target studied is homogeneous or if it is possible to take numerous readings of the same heterogeneous target. Statistically, multiple readings are expected to give correct proportions for each end-member overall. Determining the number of samples requires some knowledge of the actual measured area and is associated with the size and distribution of the target substrates. If, however, the combination of end-members in a single reading is important, ${ }^{19,20}$ a correction may be the only way to compensate for the FOV spatial non-uniformity. The method requires preparation work and a digital camera that may not be suitable for many applications. 
With the above in mind, even without the need for precise control-the knowledge of the FOV shape and position can significantly improve single or multiple reading strategies. In the first stage of this investigation it was established that the actual effective FOV is eight times smaller than that calculated from distance and lens opening angle. Moreover, in the case of the GER1500, the non-uniformity is spectrally independent which means that the FOV dimensions are applicable for the entire spectral range. This has implications relating to the scale ratio between the sampled substrate unit and the size of the FOV. Knowing the real FOV size may influence the sampling strategy in terms of distance from the target or number of repeated samplings required to achieve correct representation of the relevant study site. The FOV mapping procedure itself is relatively simple to undertake.

One of the greatest advantages of the GER1500 in respect to the FOV non-uniformity challenge is its laser guide. Its use may facilitate accurate implementation of orientation procedure by positioning the object measured at the desired distance and direction. Correct use of this positioning guide can eliminate the need for an additional referencing tool, such as a parallel mounted digital camera (particularly if multiple readings are planned). As can be expected from simple geometry, the FOV increases linearly in size with increasing distance from the target. Due to positional offset, the laser position shifts rightwards as the sensor draws further away from the target. Based on the mapping of the laser guide position, it is possible to generally predict when the strip will be on the target and aim the sensor accordingly. With this improved sampling accuracy, if multiple measurements are undertaken no further correction may be necessary. Furthermore, in further work (to be discussed in a future paper), it was possible to use the above ratio to sample specific underwater targets up to $4 \mathrm{~m}$ deep (after which it was hard to spot the laser guide). This was extremely useful and without it errors in data collection would have occurred.

The mapping of the FOV was consistent at both resolution levels used $(2 \mathrm{~cm}$ and $1 \mathrm{~cm}$ pixel size). The higher resolution mapping $(1 \mathrm{~cm} \times 1 \mathrm{~cm})$ provided a good, wavelength-dependent correction mask that was successfully used for all three colour arrangements.

Accuracy scoring used two complementary systems-the $r^{2}$ scoring and the RMSE scoring. The $r^{2}$ method is more sensitive to initial differences between spectra and thus produces a wider range of values (between 0 and 1). It is less sensitive to differences in illumination and thus produces useful comparisons between different spectral sources. However, the use of averages in its algorithm renders the $r^{2}$ method sensitive to spectral range choice and introduces bias when end-members are partially similar (for example, when end-members have common characteristics in a certain range). Conversely, RMSE is generally less sensitive to partial similarity but is sensitive to illumination variation. ${ }^{22-25}$ The illumination gap can cause the RMSE scoring to be high, although the spectral features are similar. This, however, can be rectified by normalising the average of one spectrum to the other. Given the differences in approach between the $r^{2}$ and RMSE (for example, parametric versus non-parametric), it is important to consider both methods for spectra comparisons. Methods such as spectral angle mapper (SAM) ${ }^{22,24,26,27}$ or others ${ }^{22,23,25}$ produce similar results.

The "corrected" spectra show distinct improvement over the untreated values. It is important to note that all correction procedures are done after the FOV is specifically limited to the area of the FOV strip. The error would be much higher if the sampling area included all the "expected" FOV based on the view angle and distance $(26 \mathrm{~cm} \times 26 \mathrm{~cm})$. This correction can be used when performing detailed laboratory-based studies of spectral mixing ${ }^{19,20}$ and may reduce some of the sources of unexplained variance these studies often show. ${ }^{19}$

\section{Conclusion}

The non-uniformity in the expected FOV of the spectroradiometer unit we tested is caused by the physical build of the unit (i.e. lens and light entry slit) so other units of the same make and model are expected to demonstrate similar nonuniformities. Additionally, because this spectrometer model has a laser guide with a fixed angle (and thus a fixed shift) to the FOV plane, a linear relationship can be calculated enabling practical use of this facility. With the shift known, a practical outdoor method for aiming the unit more precisely at the target is possible.

Knowledge of a sensor's FOV shape and size is crucial for correctly measuring spectral data of a heterogeneous target. The active FOV of the GER1500 is limited to about $5 \%$ of the expected area based on the simple view angle and distance from unit to target. However, because the non-uniformity is spectrally independent, correction is simplified. We have shown how the correction procedure was successfully implemented in the analysis and correction of a range of mixed spectra. In addition, knowing the shape of the actual FOV combined with the laser guide, the GER1500 can facilitate reasonably accurate operations on heterogeneous targets.

Further work is required in describing the FOV of other spectrometers using the FOV mapping suggested in this study.

\section{Acknowledgements}

We would like to thank the Natural Environment Research Council (NERC) Field Spectroscopy Facility (FSF- http://fsf. nerc.ac.uk/I who provided the equipment. The study was partly funded by EU's Dryland Research Specific Support Action (SSA) via Ben Gurion University. Additional funding was provided by Bar Ilan University who provided laboratory space and logistical support through the Interuniversity Marine Laboratory, Eilat, Israel. Finally, J. Hedley was funded by NERC under grant NE/E015654/1 


\section{References}

1. H. Holden, "Coral reef ecosystem change detection based on spatial autocorrelation of multispectral satellite data," Geoscience and Remote Sensing Symposium (2000).

2. D.G. Goodenough, A. Dyk, K.O. Niemann, J.S. Pearlman, C. Hao, T. Han, M. Murdoch and C. West, "Processing Hyperion and ALI for forest classification," IEEE Trans. Geosci. Remote Sens. 41(6), 1147 (2003). doi: 10.1109/ TGRS.2003.813214

3. M.E. Schaepman, "Spectrodirectional remote sensing: from pixels to processes," Int. J. Appl. Earth Obs. 9(2), 204 (2007). doi:10.1016/j.jag.2006.09.003

4. E.P. Green, P.J. Mumby, A.J. Edwards and C.D. Clark, Remote Sensing Ha., UNESCO, Paris, France (2000).

5. P.M. Mather and M. Koch, Computer Processing of Remotely-Sensed Images: An Introduction. John Wiley \& Sons, Chichester, UK (2004).

6. E.J. Milton, M.E. Schaepman, K. Anderson, M. Kneubühler and N. Fox, "Progress in field spectroscopy," Remote Sens, Environ. (2007). doi: 10.1016/j.rse.2007.08.001

7. E.J. Milton, "Review article: Principles of field spectroscopy," Int. J. Remote Sens. 12, 1807 (1987). doi: $\underline{10.1080 / 01431168708954818}$

8. M.D. Steven, "Correcting the effects of field of view and varying illumination in spectral measurements of crops," Precis. Agric. 5(1), 55 (2004). doi: 10.1023/B:PRAG.000001 3620.61519 .86

9. C. Gomez, P. Lagacherie and G. Coulouma, "Continuum removal versus PLSR method for clay and calcium carbonate content estimation from laboratory and airborne hyperspectral measurements," Geoderma 148(2), 141 (2008). doi: 10.1016/j.geoderma.2008.09.016

10. H. Duan, R. Ma, B. Zhang, K. Song and Z. Wang, "Determination of chlorophyll-a concentration using inverse continuum removal analysis of fluorescence peak in Lake Chagan, China," SPIE Conference Series, Vol. 6752. SPIE, Bellingham, USA (2007).

11. A. Gendrin, F. Poulet, N. Charvin, Y. Langevin and J.F. Mustard, "Combining a non linear unmixing model and the tetracorder algorithm: application to the ISM dataset, 35th Lunar and Planetary Science Conference, League City, Texas, USA, Abstract no. 1207 (2004).

12. G.P. Asner and K.B. Heidebrecht, "Spectral unmixing of vegetation, soil and dry carbon cover in arid regions: comparing multispectral and hyperspectral observations," Int. J. Remote Sens. 23(19), 3939 (2002). doi: $\underline{10.1080 / 01431160110115960}$

13. N. Dobigeon, S. Moussaoui, M. Coulon, J.-Y. Tournere and A.O Hero, "Joint Bayesian endmember extraction and linear unmixing for hyperspectral imagery," IEEE Trans. Signal Process. 57(11), 4355 (2009). doi: $10.1109 /$ TSP.2009.2025797

14. N. Keshava, "A survey of spectral unmixing algorithms," Lincoln Lab. J. 14(1), 55 (2003).
15. P.L. Rosin, “Robust pixel unmixing," IEEE Trans. Geosci. Remote Sens. 39(9), 1978 (2001).

16. A.A. MacArthur, C. MacLellan and T.J. Malthus, "The implications of non-uniformity in fields-of-view of commonly used field spectroradiometers," in Geoscience and Remote Sensing Symposium, Barcelona 2007. IEEE International, California, USA, pp. 2890-2893 (2007).

17. J. Kerekes, M.A. Glennon and R. Lockwood, “Unmixing analysis: model prediction compared to observed results," in Geoscience and Remote Sensing Symposium, 2003. IEEE International, California, USA, pp. 99-102 (2003).

18. J. Hedley, "Spectral unmixing of coral reef benthos under ideal conditions". PhD Thesis, University of Exeter, Exeter, UK (2004).

19. J. Hedley, P. Mumby, K. Joyce and S.R. Phinn, "Spectral unmixing of coral reef benthos under ideal conditions," Coral Reefs 23(1), 60 (2004). doi: 10.1007/s00338-0030354-x

20. J. Zhang, B. Rivard and A. Sanchez-Azofeifa, "Derivative spectral unmixing of hyperspectral data applied to mixtures of lichen and rock," IEEE Trans. Geosci. Remote Sens. 42(9), 1934 (2004). doi: 10.1109 / TGRS.2004.832239

21. A.A. MacArthur, C. Maclellan and T.J. Malthus, "What does a spectroradiometer see?", The Remote Sensing and Photogrammetry Society Annual Conference, Fitzwilliam College, University of Cambridge, Cambridge, UK (2006).

22. P.E. Dennison, K.Q. Halligan and D.A. Roberts, "A comparison of error metrics and constraints for multiple endmember spectral mixture analysis and spectral angle mapper," Remote Sens. Environ. 93(3), 359 (2004). doi: $\underline{10.1016 / \text { i.rse.2004.07.013 }}$

23. M.A. Pizarro and D. Fernandes, “Comparison among spectra similarities measures based on entropy, euclidian distance and correlation coefficient in Hyperspectral data," Geoscience and Remote Sensing Symposium, Boston, Massachusetts, USA, 2008. IEEE International, California, USA (2008).

24. Y. Zeng, M.E. Schaepman, B. Wu, J. Clevers and A. Bregt, "Deriving regional scale forest structural variables using eo-1 hyperion data in combination with an inverted geometric-optical model," in Proceeding of ISPRS: From Pixels to Processes, Enschede, The Netherlands (2006).

25. S.A. Robila and A. Gershman, "Spectral matching accuracy in processing hyperspectral data," in Proceedings of the International Symposium on Signals, Circuits and Systems, ISSCS 2005. IEEE International, California, USA, pp. 163-166 (2005).

26. F.A. Kruse, "Improved multispectral mapping by spectral modeling with hyperspectral signatures," in SPIE Symposium on Defense and Security, Orlando, Florida, USA. SPIE, Bellingham, USA (2007).

27. L. Junwu, R.L. King and N. Younan, "An unmixing algorithm based on vicinal information," Geoscience and Remote Sensing Symposium, 2002, IGARSS '02. IEEE International, California, USA, pp. 1453-1455 (2002). 